\title{
Optimal harvest and efficient use of septal cartilage in rhinoplasty
}

\author{
Sung Ho Yoon, \\ Cha Soo Kim, \\ Jae Wook Oh, \\ Keun Cheol Lee \\ Department of Plastic and \\ Reconstructive Surgery, Haeundae Paik \\ Hospital, Inje University College of \\ Medicine, Busan, Korea
}

\begin{abstract}
Background: Nasal septal cartilage is used to obtain favorable aesthetic and functional outcomes in rhinoplasty, but is often difficult to harvest or the harvested amount is insufficient. Therefore, the objective of this study is to introduce how to harvest septal cartilage optimally without losing and use harvested cartilage efficiently.

Methods: From March 2015 to January 2020, we tried to harvest as much septal cartilage as possible while maintaining the L-strut in 30 patients. A spreader flap and septal rotation suture were used instead of a spreader graft. Also in patients who needed a spreader graft and septal extension graft, a spreader graft was used on one side and a one-piece spreader graft combined with a septal extension graft was performed on the other side. For tip plasty, a columella septal suture was performed first. Postoperative patient satisfaction was assessed using the Rhinoplasty Outcome Examination questionnaire.

Results: No serious complications were observed. The patient satisfaction score was $50 \%$ or above in 27 patients $(90 \%)$ and less than $50 \%$ in only three patients $(10 \%)$. The average score was 81.5 points.

Conclusion: For septal cartilage deficiency, a spreader flap, the septal rotation suture, or onepiece spreader graft combined with a septal extension graft was used. The nasal tip was sufficiently rotated using the columellar septal suture technique first. These techniques made it possible to obtain good aesthetic outcomes using only septal cartilage, without harvesting other cartilage.
\end{abstract}

Keywords: Nasal cartilage / Nasal septum / Rhinoplasty

\section{INTRODUCTION}

Rhinoplasty is one of the most commonly performed cosmetic surgical procedures in Korea. When planning a rhinoplasty procedure, for a successful operation, the surgeon should use the most suitable surgical technique for each patient considering both functional and aesthetic aspects of the nose.

Generally, autografts have excellent properties in terms of

\footnotetext{
Correspondence: Keun Cheol Lee

Department of Plastic and Reconstructive Surgery, Haeundae Paik Hospital, Inje University College of Medicine, 875 Haeun-daero, Haeundae-gu, Busan 48108, Korea

E-mail: H00511@paik.ac.kr

Received September 8, 2020 / Revised February 15, 2021 / Accepted February 17, 2021
}

long-term shape retention, and their use substantially reduces the risk of surgical site infection compared to other implant materials [1]. In contrast, alloplastic materials are easy to implant, but they are more susceptible to infection than autografts, have a tendency to migrate over time, may produce an unnatural, stiff, and rigid appearance, and carry the risk of extrusion and translucency [2]. Currently, septal cartilage, conchal cartilage, and costal cartilage are the most common implant sources for autograft in rhinoplasty. Septal cartilage has several advantages over other autologous tissues and has thus been primarily used in surgery [3]. Septal cartilage is an essential tool in most primary and secondary rhinoplasty procedures, and it is an optimal material for reinforcing the support of major structures to 
reshape the nose and to improve the function of the nose; however, the amount of intraoperatively harvested cartilage is often insufficient for successful surgery [4]. Therefore, the objective of this study is to introduce how to harvest septal cartilage optimally without losing and use harvested cartilage efficiently. Here, we describe the aesthetic outcomes of our techniques.

\section{METHODS}

\section{Patients and methods}

A retrospective review was performed of the medical records of 30 patients treated from March 2015 to January 2020. During this period, 30 patients (14 men and 16 women) underwent surgery to correct flat nose (8 patients), hump nose (4 patients), short nose (4 patients), and deviated nose (14 patients). The patients ranged in age from 19 to 40 years (mean, 30 years). Ten patients were treated with a spreader flap and the septal rotation sutures instead of a spreader graft, and five patients underwent septal rotation sutures instead of a spreader graft. In 15 patients, a spreader graft was used on one side and a one-piece spreader graft combined with a septal extension graft was used on the other side, instead of the bilateral spreader graft and septal extension graft. In all patients, tip plasty was preceded by a columellar septal suture for sufficient rotation of the nasal tip in a cephalic or caudal direction as appropriate.

\section{Surgical technique}

All procedures were performed via open rhinoplasty under general or local anesthesia. Before harvesting septal cartilage, hydro-dissection was performed using a local anesthetic between the septal cartilage and the perichondrium to prevent

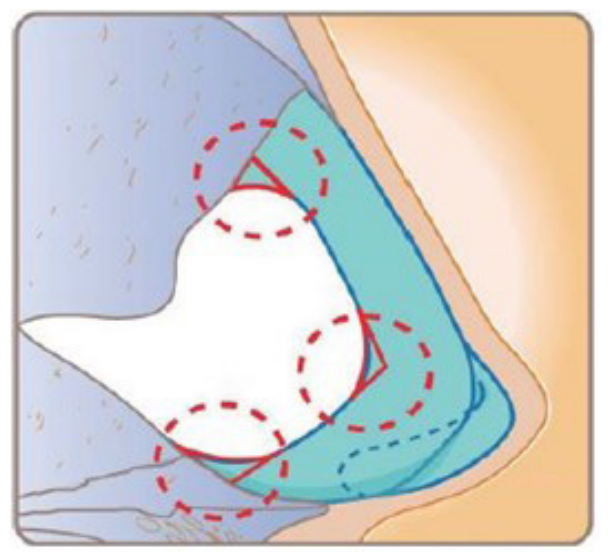

Fig. 1. A schematic diagram of septal cartilage harvest. If the incision line changes its direction, it has to be at a round angle and not a right angle (red dotted circles). The remaining small cartilage block can support the remaining L-strut. Reprinted from Jeong. Arch Plast Surg 2014;41:19-28 [4]. tearing of the mucoperichondrium. If there was an old fracture or buckling, dissection was performed meticulously while ensuring complete visibility to prevent loss of the harvested amount due to incorrect dissection or fracture during surgery. After dissection, if the incision line changes its direction, it has to be at a round angle and not a right angle. The remaining small cartilage block can support the remaining L-strut thereby improving stability of the L-strut under external stress and trauma (Fig. 1) [4]. Therefore, we could maximize the amount of harvested cartilage as long as the L-strut was maintained, and the vomer was also harvested if necessary [4].

In order to reduce the use of septal cartilage, we used the septal rotation suture and a spreader flap instead of a spreader graft depending on the patient's condition. In patients who needed bilateral spreader grafting and a septal extension graft, we reduced the use of cartilage by implanting two graft pieces, instead of the traditional three pieces; specifically, we used a spreader graft on one side and a one-piece spreader graft combined with a septal extension graft on the other side (Fig. 2) $[5,6]$. In patients who required tip plasty, a columellar septal su-
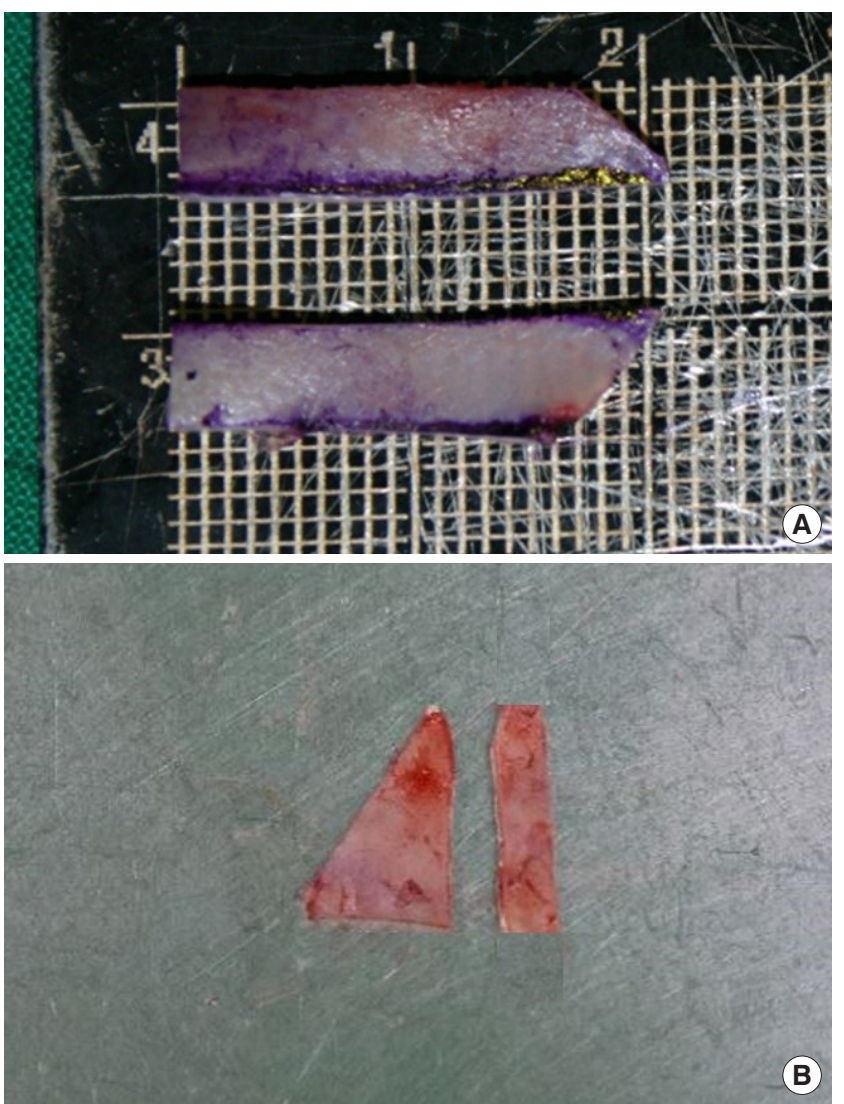

Fig. 2. Photographs of intraoperatively harvested septal cartilage. (A) Shape of spreader grafts. In patients who required a spreader graft and a simultaneous septal extension graft, a spreader graft was used on one side and a one-piece spreader graft combined with a septal extension graft was used on the other side, as shown in (B). 
ture was performed first to reduce the use of cartilage graft at the nose tip by sufficiently rotating it in a cephalic or caudal direction. Various tip suture techniques were also used to reduce the use of implanted cartilage: lateral crural spanning sutures, transdomal sutures, interdomal sutures, and intercrural sutures $[7,8]$.

\section{Aesthetic outcome assessment}

Patient satisfaction was investigated using the Korean version of the Rhinoplasty Outcome Evaluation (ROE) questionnaire 1 year after surgery (Table 1). The overall score ranges from $0 \%$ to $100 \%$, with $0 \%$ indicating "very disappointed" and $100 \%$ indicating "very satisfied." Patients with a score of $50 \%$ or more are considered as being satisfied with the outcome [9]. In addition, three plastic surgeons (JWO, SHY, Young Geum Kim

Table 1. The ROE questionnaire

\begin{tabular}{|c|c|}
\hline ROE questionnaire & Scores and answers \\
\hline \multirow{5}{*}{ Q1: Do you like how your nose looks? } & 0: Absolutely no \\
\hline & 1: A little \\
\hline & 2: More or less \\
\hline & 3: Very much \\
\hline & 4: Absolutely yes \\
\hline \multirow[t]{5}{*}{ Q2: Do you breathe well through your nose? } & 0 : Absolutely no \\
\hline & 1: A little \\
\hline & 2: More or less \\
\hline & 3: Very much \\
\hline & 4: Absolutely yes \\
\hline \multirow{5}{*}{$\begin{array}{l}\text { Q3: Do you believe your friends and people who are dear } \\
\text { to you like your nose? }\end{array}$} & 0: Absolutely no \\
\hline & 1: A little \\
\hline & 2: More or less \\
\hline & 3: Very much \\
\hline & 4: Absolutely yes \\
\hline \multirow{5}{*}{$\begin{array}{l}\text { Q4: Do you think the current appearance of your nose } \\
\text { hampers your social or professional activities? }\end{array}$} & $0:$ Always \\
\hline & 1: Frequently \\
\hline & 2: Sometimes \\
\hline & 3: Rarely \\
\hline & 4: Never \\
\hline \multirow[t]{5}{*}{ Q5: Do you think your nose looks as good as it could be? } & 0: Absolutely no \\
\hline & 1: A little \\
\hline & 2: More or less \\
\hline & 3: Very much \\
\hline & 4: Absolutely yes \\
\hline \multirow{5}{*}{$\begin{array}{l}\text { Q6: Would you undergo surgery to change the appearance } \\
\text { of your nose or to improve your breathing? }\end{array}$} & 0: Certainly yes \\
\hline & 1: Very likely yes \\
\hline & 2: Possibly yes \\
\hline & 3: Probably no \\
\hline & 4: Certainly no \\
\hline
\end{tabular}

ROE, Rhinoplasty Outcome Examination.
[Haeundae Paik Hospital, Inje University College of Medicine, Busan, Korea]) who did not participate in the operations compared a total of six photographs including the frontal view, lateral view, and worms' eye view taken before and after surgery and classified the results as excellent, good, fair, or poor.

\section{RESULTS}

Of the 30 patients (14 men and 16 women) included in this study, 25 patients (73\%) underwent primary rhinoplasty and five patients (27\%) underwent secondary rhinoplasty. Twentyseven patients $(90 \%)$ were satisfied with the operation, with an ROE score of $50 \%$ or higher, and only three patients (10\%) had a score less than $50 \%$ (Table 2). The three plastic surgeons rated the outcome as excellent in seven patients (23.3\%), good in ten patients (33.3\%), fair in nine patients (30.0\%), and poor in four patients (13\%). The mean follow-up period was 18 months and no serious complications were observed (Table 3, Figs. 3-5).

\section{DISCUSSION}

Rhinoplasty has become a common procedure as a result of economic growth and the increasing popularity of plastic surgery. Accordingly, the cosmetic expectations of patients are also rising, and plastic surgeons' incessant efforts to meet these ex-

Table 2. Distribution of patient satisfaction according to ROE scores

\begin{tabular}{lc}
\hline Total ROE score (\%) & Patients \\
\hline Score $<50$ & 27 \\
Score $\geq 50$ & 3 \\
\hline
\end{tabular}

Patients with a score of $50 \%$ or more can be considered to be satisfied with their aesthetic results. The average score was $81.5 \%$.

ROE, Rhinoplasty Outcome Examination.

Table 3. Classification as comparison of preoperative and postoperative images

\begin{tabular}{lc}
\hline Variable & No. (\%) \\
\hline Surgeon's opinion ${ }^{2)}$ & $30(100)$ \\
Excellent & $7(23.3)$ \\
Good & $10(33.3)$ \\
Fair & $9(30.0)$ \\
Poor & $4(13.3)$ \\
Complications & \\
No complications & $28(93.3)$ \\
Nasal mucosa tearing & $2(6.7)$ \\
\hline
\end{tabular}

This result was classified as excellent, good, fair, and poor and postoperative complications based on a comparison of six preoperative and postoperative images including the frontal view, lateral view, and worms' eye view.

a)The average values of the three plastic surgeons' assessment results. 

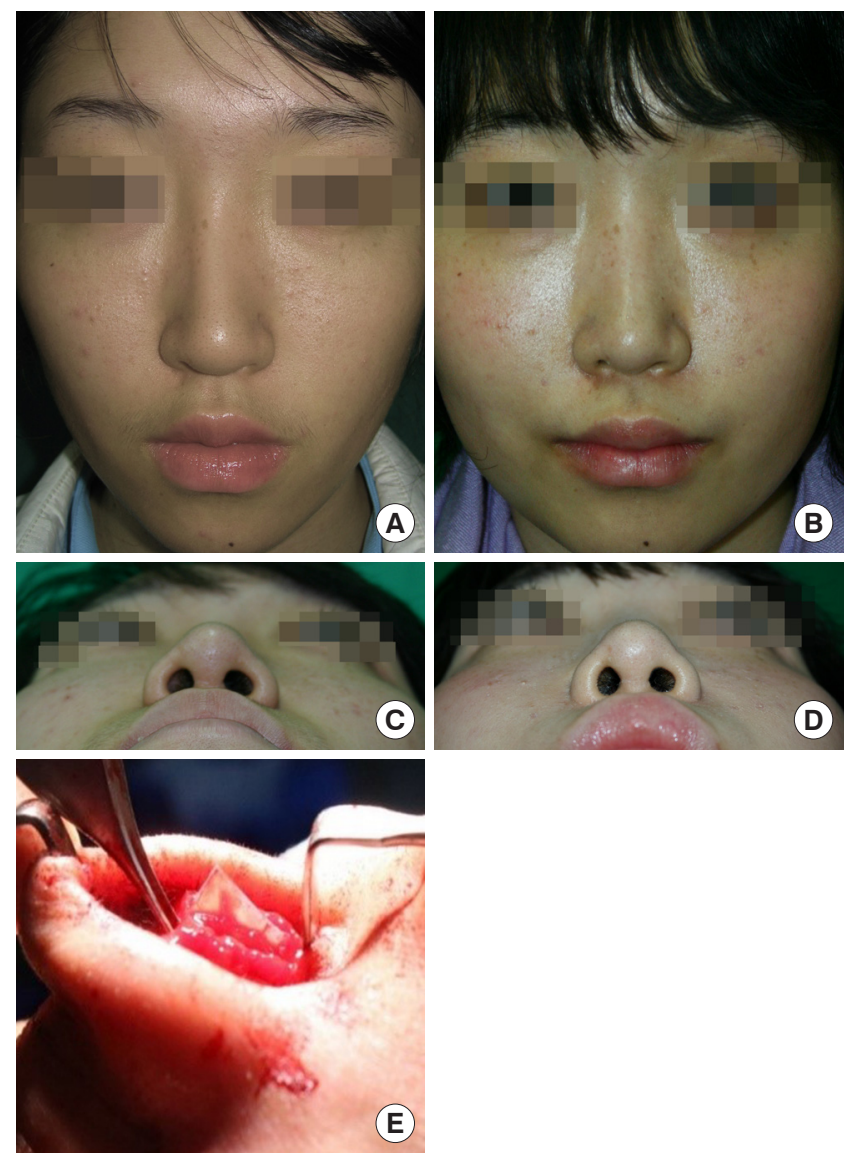

Fig. 3. A 28-year-old woman with a bulbous tip and mild deviation of the nasal root. (A) Preoperative frontal view. (B) Postoperative frontal view. (C) Preoperative worm's eye view. (D) Postoperative worm's eye view. (E) Intraoperative photograph showing a spreader graft on one side and a one-piece spreader graft combined with a septal extension graft on the other side. We inserted a silicone implant to improve the nasal height and used a columella septal suture and a transdomal suture instead of a tip graft for tip projection.

pectations have led to significant advances and innovations in both the surgical process and outcomes.

It is now difficult to meet patients' expectations with simple augmentation rhinoplasty alone. Therefore, surgical techniques designed to be tailored to individual patient's specific needs are necessary, and the use of various types of implants is also increasing. Autografts are known to be superior to alloplastic materials and other alternatives for reducing postoperative complications and improving functional and aesthetic outcomes [10].

Currently, the most common autologous tissue sources are septal cartilage, conchal cartilage, and costal cartilage [10]. Conchal cartilage is frequently used to obtain a small graft. It is advantageous in that the donor site can be sutured easily and scarring is almost invisible; furthermore, unlike costal cartilage, it rarely warps and is flexible and elastic. However, unlike septal cartilage, it requires an additional surgical procedure to harvest
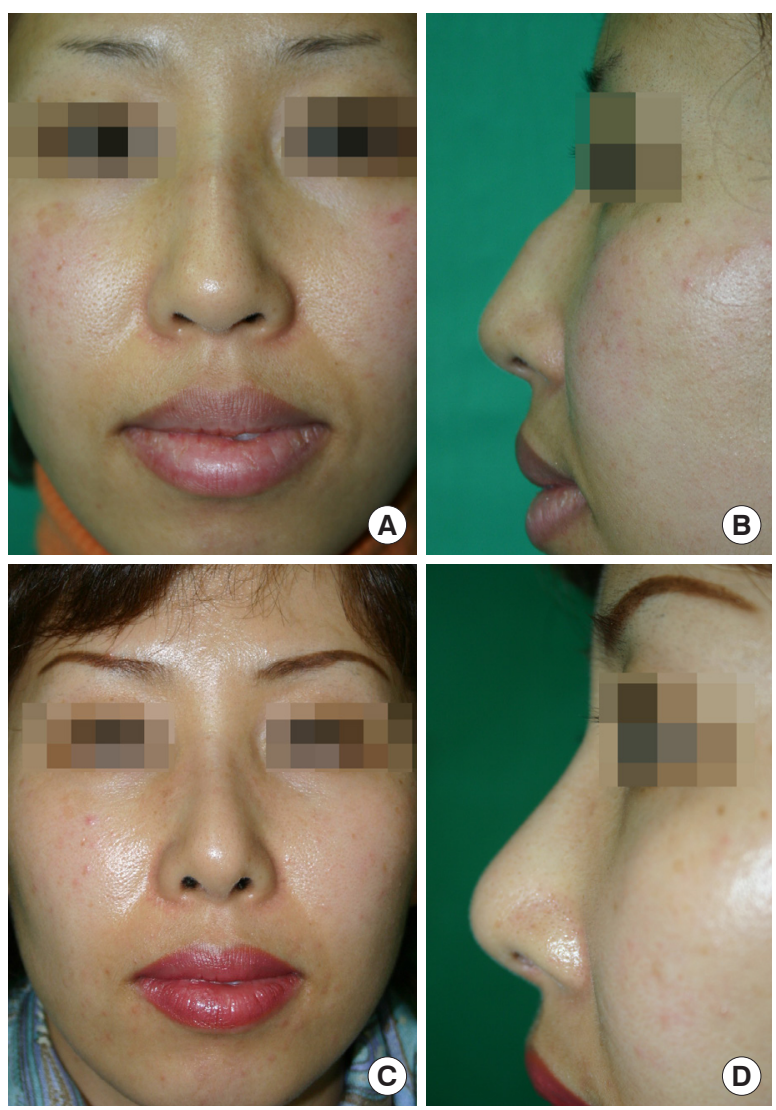

Fig. 4. A 38-year-old female patient with hump nose and drooping nose. (A) Preoperative frontal view. (B) Preoperative lateral view. (C) Postoperative frontal view. (D) Postoperative lateral view. Nasal deviation was corrected by lateral osteotomy using a 2-mm lateral osteotome. Additionally, the shape was refined with humpectomy and rasping. Alloderm was used on the nasal dorsum.

from a different location other than the surgical site, and the shape of the ear should be taken into consideration, which may restrict sufficient harvesting. It is mostly curved and lacks a flat surface, it is inconvenient to manipulate. In addition, it tends to be brittle [11].

Unlike other types of cartilage, costal cartilage is very useful when extensive correction is required due to severe nasal deformity, as an abundance of cartilage can be harvested. However, it requires an additional procedure for harvesting and carries the risk of prominent chest scarring and pneumothorax. In particular, the shape of the nose may change due to warping after implantation, and ossification may occur in some patients [12].

Septal cartilage is easier to harvest than other types of cartilage, it leaves no additional scars, and it has properties similar to those of the cartilaginous structures of the nose; thus, it is currently one of the most common graft sources in rhinoplasty. However, as most Asians have smaller noses than individuals of other races, the amount of available cartilage is limited and the amount necessary for a satisfactory outcome is relatively large 

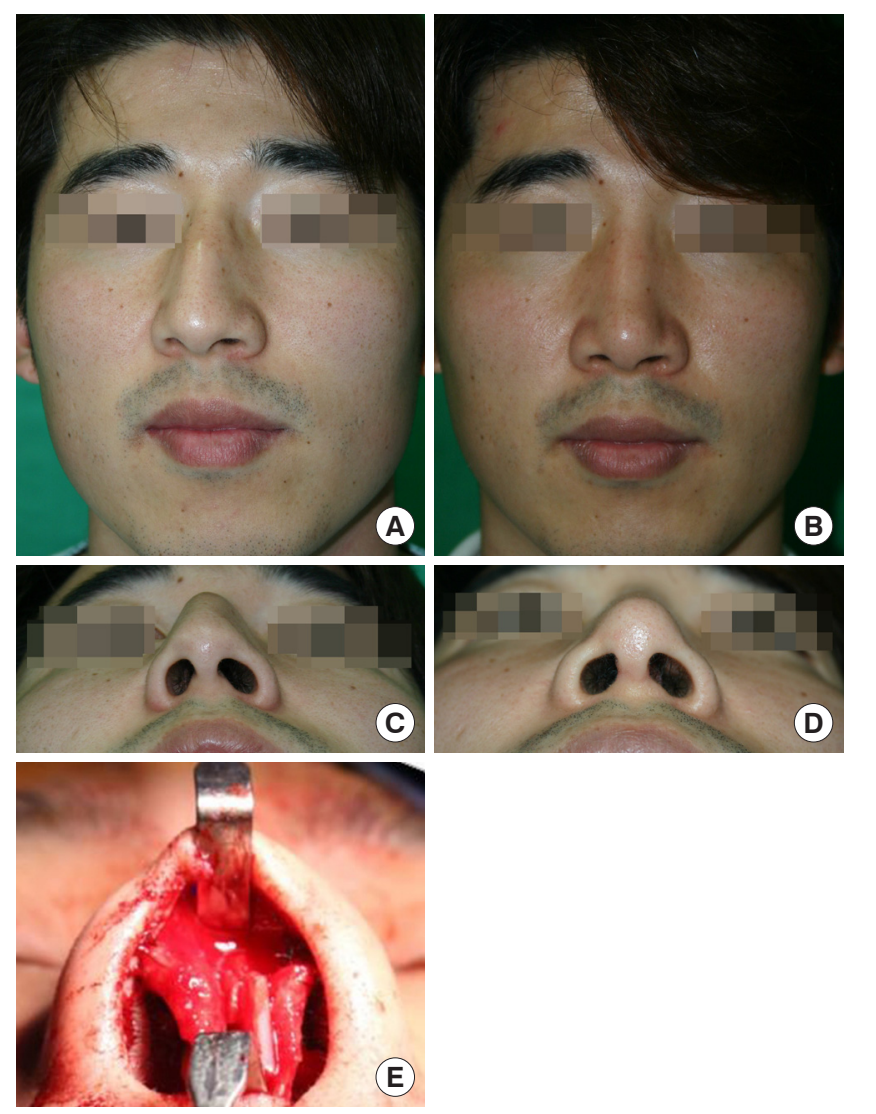

Fig. 5. A 35-year-old man with hump nose and deviated nose. (A) Preoperative frontal view. (B) Postoperative frontal view. (C) Preoperative worm's eye view. (D) Postoperative worm's eye view. (E) Intraoperative photograph. A spreader graft was used on one side and a one-piece spreader graft combined with a septal extension graft was used on the other side. Humpectomy and lateral ostectomy using a 2-mm lateral osteotome were performed. A septal rotation suture was used to improve deviation of the upper lateral cartilage.

in most cases [13]. Harvesting as much cartilage as possible and using it in the most efficient possible manner are considered to be essential.

Therefore, we sought to develop a method to safely harvest as much septal cartilage as possible and use it with optimal efficiency. To maximize harvesting of the cartilage, we performed full hydro-dissection between the cartilage and the perichondrium with a local anesthetics to prevent tearing of the mucoperichondrium of the septum before harvesting the septal cartilage. In particular, if an old fracture or buckling was present, dissection was performed meticulously under complete visualization to prevent reduction in the amount of harvested cartilage due to incorrect dissection or fracture during surgery. After dissection, if the incision line changes its direction, it has to be at a round angle and not a right angle. The remaining small cartilage block can support the power of the remaining L-strut a cartilage [4]. These small cartilage blocks made the L-strut withstand external stress and trauma more strongly; thus, in some patients, a relatively large amount of cartilage could be safely harvested while the minimum L-strut was maintained, and the vomer was included in harvesting if necessary [4].

In addition, we used various surgical methods to replace the use of septal cartilage. First, spreader flaps, instead of spreader grafts, and septal rotation sutures were used according to the patient's condition. Second, in patients who needed a spreader graft and a septal extension graft, a spreader graft was used on one side, and a one-piece spreader graft combined with a septal extension graft was used on the other side. Third, in patients who needed tip plasty, the columellar septal suture was used first to rotate the tip of the nose to the cephalic or caudal direction as appropriate in an attempt to reduce the amount of the implanted graft. Furthermore, we used various tip suture techniques including lateral crural spanning sutures, transdomal sutures, interdomal sutures, and intercrural sutures $[7,8]$.

Spreader flap can compensate for the disadvantages of a spreader graft, such as movement of the graft material and dropping of harvested septal cartilage into the mucoperiochondrial pocket, as well as reducing the required amount of septal cartilage. It is easy to use, effective for airway preservation, and has the same effect as the spreader graft in terms of preventing an inverted $\mathrm{V}$ deformity after hump resection. Therefore, we used spreader flap for patients with features such as insufficient septal cartilage, high and narrow nasal dorsum, a short nasal bone, weak middle vault, thin nose skin, prominent hump, positive Cottle test before surgery, high upper lateral cartilage, and tension nose [5]. A septal rotation suture was used only when septal deviation was not too severe to be corrected without additional procedures. In some patients, a spreader flap and a septal rotation suture were used simultaneously.

If a spreader graft and a septal extension graft are required at the same time due to the patient's condition, it is conventional to use a total of three graft pieces, including two pieces of spreader graft and one piece of septal extension graft. However, we used a spreader graft on one side, and a one-piece spreader graft combined with a septal extension graft on the other side. In other words, we could achieve the same effect as the threepiece method using only two pieces of septal cartilage.

It is important to appropriately use various suture techniques in order to reduce the required amount of septal cartilage for tip graft. As Asians have thicker skin and weaker cartilaginous structures than Caucasians, it can be difficult to achieve sufficient tip projection as desired by the surgeon and the patient with suture techniques alone. However, in patients who required tip plasty, we tried to reduce the required amount of nasal tip graft for appropriate tip projection through sufficient ro- 
tation of the nasal tip in a cephalic or caudal direction using a columellar septal suture, and we used various tip suture techniques such as lateral crural spanning sutures, transdomal sutures, interdomal sutures, and intercrural sutures [7,8].

In conclusion, septal cartilage is an important material in rhinoplasty. Therefore, whether septal cartilage can be safely harvested and used most appropriately can be a key determinant of the success or failure of rhinoplasty. In this study, we were able to achieve aesthetically successful outcomes using only septal cartilage without the use of other cartilage. In other words, we believe that if septal cartilage is safely harvested as much as possible and used with optimal efficiency, satisfactory rhinoplasty can be performed.

\section{NOTES}

\section{Conflict of interest}

No potential conflict of interest relevant to this article was reported.

\section{Ethical approval}

The study was approved by the Institutional Review Board of Haeundae Paik Hospital (IRB No. 2020-07-034-002) and performed in accordance with the principles of the Declaration of Helsinki. Written informed consent was waived.

\section{Patient consent}

The patients provided written informed consent for the publication and the use of their images.

\section{ORCID}

Sung Ho Yoon https://orcid.org/0000-0003-4765-2308

Cha Soo Kim

Jae Wook Oh https://orcid.org/0000-0002-2451-3025

Keun Cheol Lee https://orcid.org/0000-0001-8829-0935 https://orcid.org/0000-0003-0553-0900

\section{Author contribution}

Conceptualization: SHY. Formal analysis: JWO. Project administration: KCL. Writing - original draft: CSK, KCL. Writing - review \& editing: CSK. Approval of final manuscript: all authors.

\section{REFERENCES}

1. Ishii CH. Current update in Asian rhinoplasty. Plast Reconstr Surg Glob Open 2014;2:e133.

2. Sajjadian A, Naghshineh N, Rubinstein R. Current status of grafts and implants in rhinoplasty: part II. Homologous grafts and allogenic implants. Plast Reconstr Surg 2010;125:99e-109e.

3. Sancho BV, Molina AR. Use of septal cartilage homografts in rhinoplasty. Aesthetic Plast Surg 2000;24:357-63.

4. Jeong JY. Obtaining maximal stability with a septal extension technique in East Asian rhinoplasty. Arch Plast Surg 2014;41: 19-28.

5. Gruber RP, Park E, Newman J, Berkowitz L, Oneal R. The spreader flap in primary rhinoplasty. Plast Reconstr Surg 2007; 119:1903-10.

6. Paik MH, Chu LS. Correction of short nose deformity using a septal extension graft combined with a derotation graft. Arch Plast Surg 2014;41:12-8.

7. Lee KC, Kwon YS, Park JM, Kim SK, Park SH, Kim JH. Nasal tip plasty using various techniques in rhinoplasty. Aesthetic Plast Surg 2004;28:445-55.

8. Gruber RP, Weintraub J, Pomerantz J. Suture techniques for the nasal tip. Aesthet Surg J 2008;28:92-100.

9. Kotzampasakis D, Mantalos P, Kotzampasakis S, Danias N, Nikolopoulos T. Assessment of aesthetic results of 100 patients who underwent rhinoplasty-rhinoplasty outcome evaluation. Plast Reconstr Surg Glob Open 2017;5:e1404.

10. Sajjadian A, Rubinstein R, Naghshineh N. Current status of grafts and implants in rhinoplasty: part I. Autologous grafts. Plast Reconstr Surg 2010;125:40e-49e.

11. Jovanovic S, Berghaus A. Autogenous auricular concha cartilage transplant in corrective rhinoplasty: practical hints and critical remarks. Rhinology 1991;29:273-9.

12. Park JH, Jin HR. Use of autologous costal cartilage in Asian rhinoplasty. Plast Reconstr Surg 2012;130:1338-48.

13. Kim TK, Jeong JY. Surgical anatomy for Asian rhinoplasty. Arch Craniofac Surg 2019;20:147-57. 